\title{
Design of the SC-FDE System Applied in Broadband HF Burst Communication
}

\author{
Boyuan $\mathrm{Xu}^{1, \mathrm{a}}$, Zhiqiang Ye $\mathrm{e}^{1, \mathrm{~b}}$, Yue $\mathrm{Ma}^{1, \mathrm{c}}$ and Lifu Wu ${ }^{1, \mathrm{~d}}$ \\ ${ }^{1}$ Academy of Broadcasting Science State Administration of Radio, Film \&Television, China \\ axuboyuan@abs.ac.cn, byezhiqiang @abs.ac.cn, cmayue @abs.ac.cn, wulifu@abs.ac.cn
}

Keywords: SC; FDE; Synchronization; Broadband HF communication

\begin{abstract}
The application of FDE technique makes single carrier modulation a valuable alternative resisting multiple-path in broadband communication. The paper shows the principle of SC-FDE and propose a scheme applied in broadband HF channel in burst communication system. At last the paper shows the performance of scheme system under the DRM criterion channel.
\end{abstract}

\section{Introduction}

Recent years SC-FDE (Single-Carrier Frequency Domain Equalization) is more and more widely used by people owing to its advantages of low PARR (Peak-to average Ratio Requirements) and less sensitive to the frequency offset compared to OFMD (Orthogonal Frequency Division Multiplexing) system. The technology of frequency domain equalization is proposed to less down calculation of traditional way of timing equalization in SC system so that the occupied bandwidth can be raised. The paper provides a scheme with the SC-FDE system to complete the high data rate transmission under HF channel. Also gives the performance of SC-FDE system under the DRM criterion channels.

\section{SC-FDE System Design}

Principle of SC-FDE System. SC-FDE and OFDM are closed and have the similar means of signal processing. Fig. 1 shows the system flow of SC-FDE, the sending part firstly adopts technology of bit-to-symbol mapping, then the CPI module, and at last data frames are send through the channel to transmit after shaped filtering. At the receiving part, the UW in each data block is used to estimate the characteristics of the channel and get the equalizing coefficients. In the receiver, firstly data are sent into SYN module, this module is used to generate the frame data to generate the equalization. Then the data is converted to time domain by the IFFT module and UW is removed. At last the de-mapping and decision module generate the de-mapping and decision. [1]

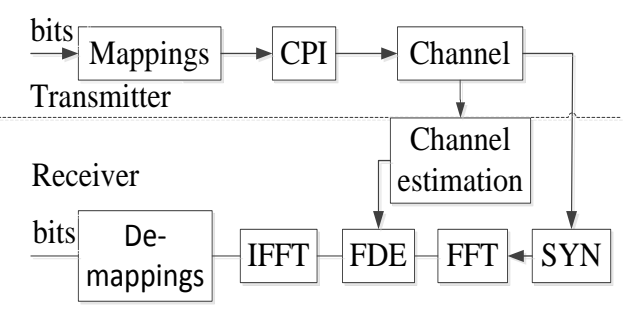

Figure 1. The structure of the SC-FDE

At las the output symbols of the de-mapping and decision module in time domain can be described: [2] [3]

$$
z_{n}=\frac{1}{N} \sum_{k=0}^{N-1} W_{k} Y_{k} H e^{j \frac{2 \pi}{N} k n}+\frac{1}{N} \sum_{k=0}^{N} W_{k} V_{k} e^{j \frac{2 \pi}{N} k n}
$$

Design of the Data Frame Structure. The design adopts the 2UW frame structure, which is shown in the Fig. 2. The burst frame lasts 1s with a frame head and 24 data blocks. The frame head includes double training symbols, and each data block consists two UW with one is at head the 
other is at last. Thus each data (D) has two UW at the front of itself. The first is used as CP like OFDM system, while the second is for channel estimation.

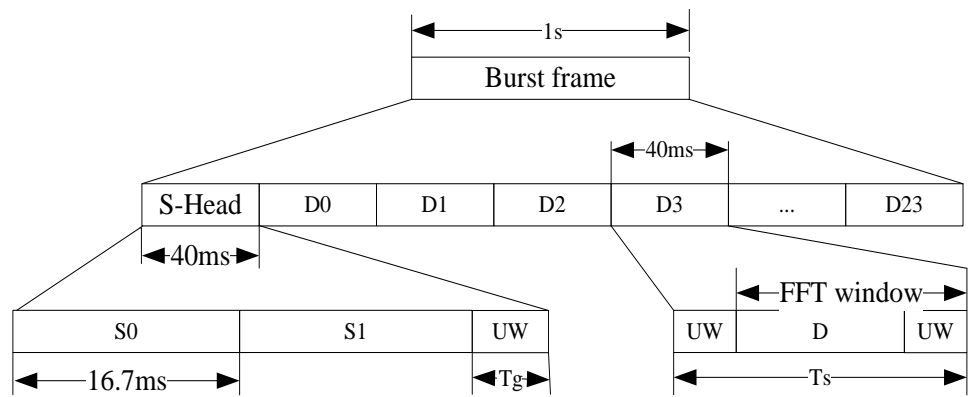

Figure 2. Data frame structures of SC-FDE

On the ideal condition UW should have the feature of constant-amplitudein the frequency domain because of its function of channel estimation, so that it can produce relative stable frequency response to test the channel characteristics at frequency points. This paper adopts the Zadoff-Chu, which has an outstanding of the constant amplitude in time and frequency domain. It can be described as follows:

$$
\begin{aligned}
& C(k)=\exp \left[j 2 \pi \frac{M}{N}\left(\frac{k(k+1)}{2}+q k\right)\right], k=0,1, \ldots N-1 \text { (N is odd.) } \\
& C(k)=\exp \left[j 2 \pi \frac{M}{N}\left(\frac{k^{2}}{2}+q k\right)\right], k=0,1, \ldots N-1 \text { ( } \mathrm{N} \text { is even.) }
\end{aligned}
$$

In which $\mathrm{N}$ is the length of sequences, and $\mathrm{q}$ is arbitrary integer.

\section{Principle of the Frequency Domain}

The precondition of adopting the FDE is the data block transmission by adding UW to realize the conversion from linear convolution to cycle convolution We can consider it that the equalizer is used to convergent the extended energy of symbol transmitting to the its own time slot. It is equal to invert a filter to make it with the sub-channel to have characteristics of banner and liner phase. Fig. 3 shows the flow of FDE, in which y $(\mathrm{k})$ are symbols received and $\mathrm{Y}(\mathrm{k})$ are the in the frequency domain. $\mathrm{Z}(\mathrm{k})$ are the multiplication of $\mathrm{Y}(\mathrm{k})$ and $\mathrm{W}(\mathrm{k})$, and $\mathrm{Z}(\mathrm{k})$ are $\mathrm{z}(\mathrm{k})$ by FFT.

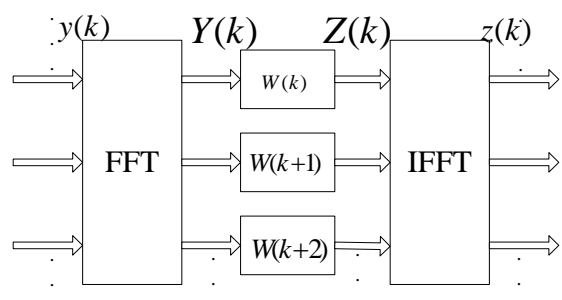

Figure 3. Structure of FDE

The technology of FDE contains liner equalization and nonlinear equalization, and it is divided by the structure of equalizer, that is whether the output is used for feedback. Liner equalization includes zero forcing ZF and MMSE, while nonlinear equalization is mainly the decision feedback equalization with the shortcoming of error propagation. This paper simulates the SC-FDE adopting the liner equalization including ZF and MMSE. The equalizing coefficients with ZF are: [4]

$$
W(z)=\frac{1}{H(z)},(k=0,1 \ldots n-1)
$$

And using MMSE are: 


$$
W(k)=\frac{H^{*}(k) \sigma_{S}^{2}}{|H(k)|^{2} \sigma_{S}^{2}+\sigma_{N}^{2}}=\frac{H^{*}(k)}{|H(k)|^{2}+\frac{\sigma_{N}^{2}}{\sigma_{S}^{2}}}
$$

The other kind of good performance equalization method is DFE. With this method frequency equalization part is as the same of the above liner equalization, while the feedback part adopts the traditional transversal filters.

By the DFE method is:

$$
W_{l}=\frac{\sigma^{2}}{M} \sum_{l=0}^{M-1} \frac{\left|F_{l}\right|^{2}}{\sigma^{2}+\left|H_{l}\right|^{2}}
$$

Fig. 4 shows the comparison performance of the three kind of method.

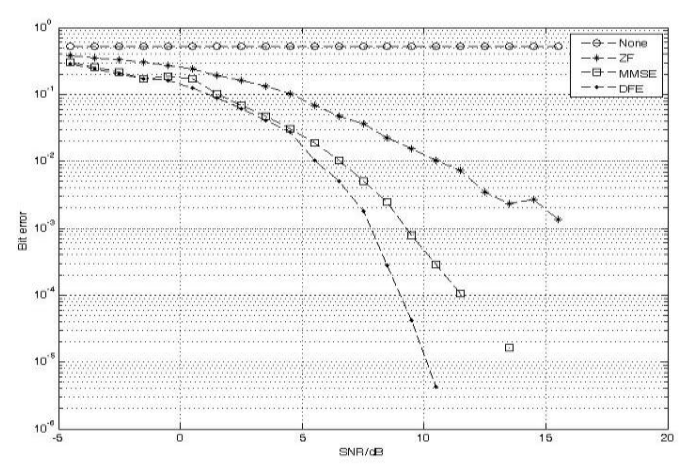

Figure 4. Constellation map with NONE equalization and MMSE

\section{Design of the Synchronization Module}

The synchronization module is at the head of the receiver, and it is used to capture the frame data out from noise, then find the accurate position of the frame-head into equalizer. At the same time, the synchronization has another function of compensation for the frequency offset because of the carrier frequency offset or dopper-shift. Fig. 5 shows the structure of the synchronization scheme in this design. The synchronization module is consisted of three parts including early capture, precise synchronization and frequency synchronization. First a capture module is used to give a judgment that whether the data arrives or not. IF the data received, capture module will send the data into the following timing synchronization and frequency offset module. Finally frame-data will be sending into the equalization module after all the three processes at last. [5]

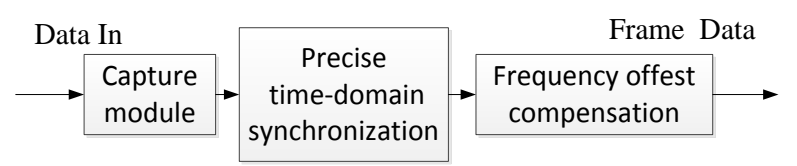

Figure 5. Structure of synchronization in SC-FDE

Early Capture Module. Early capture aims at deciding whether the frame data arrives or not. This design adopts the method of double slipping window detection based on training symbols which has a judgment of the cross-correlation value between data in the two windows. The judging value $M$ can be defined as:

$$
M(n)=|P(n)|^{2} / R(n)^{2}
$$

where the cross-correlation is:

$$
P(n)=\sum_{n=0}^{N_{S}-1} y^{*}(n) y\left(n+N_{S}\right)
$$

and energy of data in one window is: 


$$
R(n)=\sum_{n=0}^{N_{S}-1}\left|y\left(n+N_{S}\right)\right|^{2}
$$

Precise Timing Synchronization Modules. Early capture is used to judge the arriving position of data-head, while the precise synchronization module should reversely determine accurate position of the data frame-head. SC system is comparable sensitive to timing offset, while OFDM system needs to be eyed in frequency offset.

Table 1 The parameters of DRM channel 3

\begin{tabular}{|c|c|c|c|c|}
\hline \multicolumn{2}{|c|}{ Channel No.2: Rice with delay } & \multicolumn{2}{c|}{ Good typical/moderate bad } & MF HF \\
\hline parameters & Path1 & Path2 & Path3 & Path4 \\
\hline Delay(ms) & 0 & 0.7 & 1.5 & 2.2 \\
\hline Gain & 1 & 0.7 & 0.5 & 0.25 \\
\hline Doppler shift(Hz) & 0.1 & 0.2 & 0.5 & 1 \\
\hline Doppler spread(Hz) & 0.1 & 0.5 & 1 & 2 \\
\hline
\end{tabular}

In this design a method of judging the cross-correlation value between data in a certain length and the training symbols saved in local is adopted, and the corresponding position of the peak value can be defined as the data frame-head. Fig. 6 shows the simulation result of peak position under the DRM standard channel 3 with 4 paths of which the parameters are listed in Table 1.

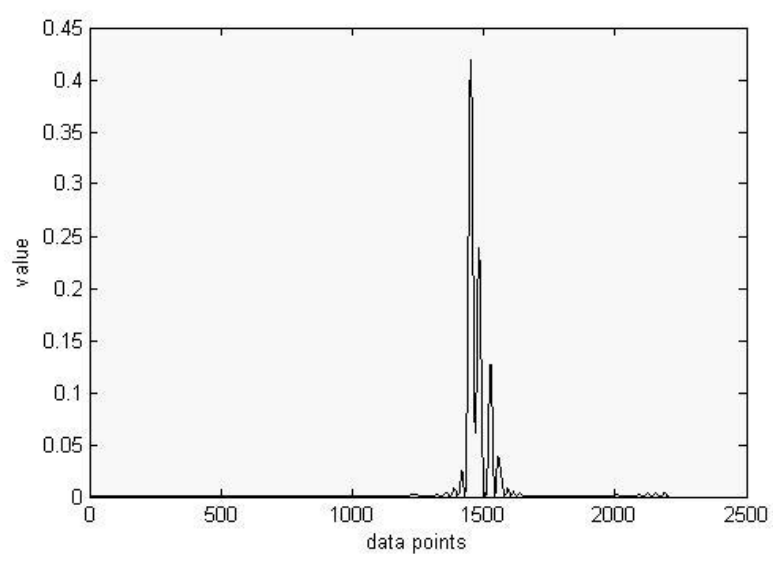

Figure 6. Simulation result when first peak is the energy peak

Frequency Offset Compensation. Carrier frequency offset is caused by the difference between transmitter and receiver carrier wave frequency in SC-FDE system. Frequency offset is need to be get and compensation while the phase offset could be correct in the following equalization module. Schmidle-Cox propose a method based on the structure of [A, A] shown in Fig. 7 to get frequency offset by compute the cross-correlation of the two training symbols. [6] [7]

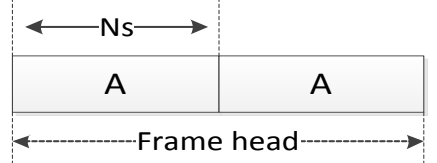

Figure 7. The structure of $[\mathrm{A}, \mathrm{A}]$

Assuming the first training symbol is expressed as:

$$
y^{\prime}(n)=y(n) \times \exp \left(j \frac{2 \pi(I+\varepsilon) n}{N_{S}}\right)
$$

and the second is: 


$$
y^{\prime}\left(n+N_{S}\right)=y\left(n+N_{S}\right) \times \exp \left(j \frac{2 \pi(I+\varepsilon)\left(n+N_{S}\right)}{N_{S}}\right)
$$

Where the frequency is divided into two parts including decimal ( $\varepsilon$ )and integer(I), and the result of the conjugate operation is:

$$
y^{\prime}(n)^{*} \times y^{\prime}\left(n+N_{S}\right)=|y(n)|^{2} \exp (j \times 2 \pi(I+\varepsilon))
$$

Then the phase angle of the result is the decimal frequency offset.

$$
\varepsilon=\frac{1}{2 \pi} \arg \left[\sum_{n=0}^{N_{S}-1} y^{\prime}(n)^{*} \times y^{\prime}\left(n+N_{S}\right)\right]
$$

Then combine the integer and decimal frequency offset, and make up for the frame data received by the frequency offset value.

\section{Simulation Results}

This simulation adopts the data frame of $2 \mathrm{UW}$ structure shown in Fig. 2 under DRM criterion channels whose parameters are listed in Table 3.

Table 2 Simulation parameters of SC-FDE

\begin{tabular}{|c|c|c|c|c|}
\hline \multicolumn{2}{|c|}{ Channel No.2: Rice with delay } & \multicolumn{2}{|c|}{ Good typical/moderate bad } & MF HF \\
\hline parameters & Path1 & Path2 & Path3 & Path4 \\
\hline Delay(ms) & 0 & 1 & & \\
\hline Gain & 1 & 0.5 & & \\
\hline Doppler shift(Hz) & 0 & 0 & & \\
\hline Doppler spread(Hz) & 0 & 0.1 & & \\
\hline \multicolumn{2}{|c|}{ Channel No.4: CCIR Poor } & \multicolumn{2}{|c|}{ Good typical/moderate bad } & MF HF \\
\hline parameters & Path1 & Path2 & Path3 & Path4 \\
\hline Delay(ms) & 0 & 2 & & \\
\hline Gain & 1 & 1 & & \\
\hline Doppler shift(Hz) & 0 & 0 & & \\
\hline Doppler spread(Hz) & 1 & 1 & & \\
\hline
\end{tabular}

\begin{tabular}{|c|c|}
\hline Band-width(KHz) & 12 \\
\hline Mapping mode & QPSK \\
\hline Aggregate data Rate(Kbps) & 19.8 \\
\hline Data block length Ts (ms) & 40 \\
\hline UW length Tg (ms) & 6.7 \\
\hline Frame Head $(\mathrm{ms})$ & 40 \\
\hline Valid data Rate $(\mathrm{Kbps})$ & 12.288 \\
\hline
\end{tabular}

Table 3 Parameters of the DRM channels

Fig. 8 shows the simulation results with the parameters which are listed in Table 2. It can be concluded that the system designed shows in a good performance and the scheme provided can be applied in broadband HF channel. 


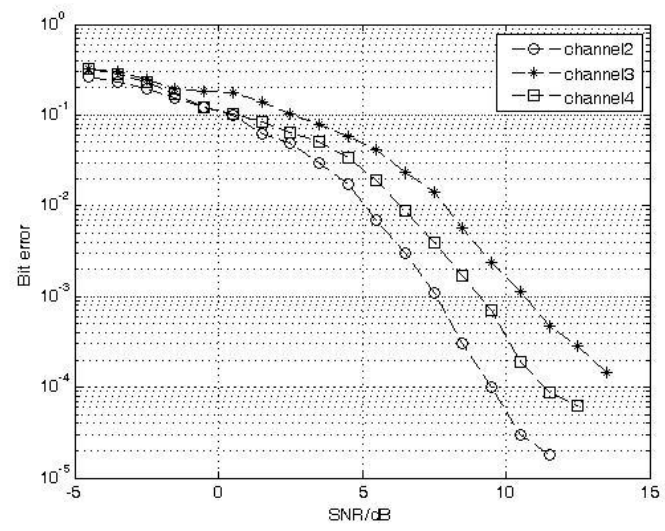

Figure 8. Simulation result under parameters

\section{References}

[1] David F, Sirikiat Lek A, Anader B-S (2002) Frequency domain equalization for single-carrier broadband wireless systems. IEEE Commun Mag 9:58-66.

[2] Qing Zhang, Tho Le-Ngoc. Channel-estimate-based frequency-domain equalization (CE-FDE) for broadband single-carrier transmission. Wireless Communication and Mobile Computing. 2004(4). pp: 449-461.

[3] M.Huemer, A. Koppler, R.Weigel, et al. Areview of cyclically extended single carrier transmission with frequency domain equalization for broadband wireless transmission. Eruopean Transactions on Telecommunications, 2003. Vol.14: 329 341.

[4] Martin V.Clark. Adaptive Frequency-Domain Equalization and Diversity Combining for Broadband Wireless Communications. IEEE Journal on Selected Areas in Communications. Oct.1998, Vol.16. pp:1385-139

[5] Timothy M. Schmdl. T M, Cox D C. Robust frequency and timing synchronization for OFDM [J]. IEEE Transactions on Commun, 1997, 45(12); 1613-1621.

[6] David C.Chu. Polyphase Codes with Good Periodic Correlation Properties. IEEE Transactions on Information theory. Jul.1972. pp: 531-532.

[7] R.L.Frank, S.A.Zadoff. Phase Shift Pulse Codes With Good Periodic Correlation Properties. IRE Transactions on Information theory. Oct.1962. pp: 381-382.

[8] Y.G. Sun, W.L. Li, D.S. Dong, X. Mei, H.Y. Qiang, "Dynamics Analysis and Active Control of a Floating Crane", Tehnicki Vjesnik-Technical Gazette, vol.22, no. 6, pp. 1383-1391, 2015. 